\title{
[Industrial paper] Application for Optimization of Control Parameters for Multi-body and Hydraulics System by using FMU
}

\author{
Nobumasa Ishida ${ }^{1}$ Hideyuki Muramatsu ${ }^{2}$ \\ ${ }^{1}$ Dassault Systèms K.K., Japan, Nobuma sa . I SH IDA@ 3ds . com \\ ${ }^{2}$ Dassault Systèms K.K., Japan, Hi deyuki . MURAMATSU@ 3ds . com
}

\begin{abstract}
We developed a simulation environment using FMU (Function Mockup Unit) as performance prediction method in the early stage of development.

In this environment, hydraulic controller parts are modeled in Dymola which is one of 1D simulation tools based on Modelica language and controlled mechanics parts are modeled in Simpack which is multibody simulation tool. We created a total system model connected to hydraulic controller parts and mechanics parts by using FMI (Function Mockup Interface). Hydraulics and controller parts are converted to FMU by Dymola, and are incorporated in Simpack mechanical model.
\end{abstract}

Furthermore, we applied the system model optimization to control parameters by using Isight.

Keywords: 1D-Simulation, Multibody, Optimization 、 FMU、Dymola、Simpack、Isight

\section{Introduction}

Recently, in the manufacturing industry, shortening the development cycle and reducing the cost of product development has an important issue, so that it is required to develop an efficient method for the development.

In the product development cycle before, prototyping was essential for production and testing to performance review. Nowadays, though, thanks to improvement of simulation accuracy and calculation environment, simulation can work as virtual testing and performance review, which leads to the reduction of trial production and physical testing cost.

However, as high precision simulation needs a lot of detailed physical properties and detailed FE model, it is difficult to apply this approach to the early stage of development in terms of complexity of model creation and calculation cost.

Further, products have been more complex system than before including many components and controller.
Therefore, we need to consider whole system level to evaluate their performance. However, there are many different simulation tools that are used in each component department and parts supplier.

When creating whole system model, each modeling tool has their own interface to connect other modeling tools. Since the interface has a specification, it takes time and effort to learn and create whole system model.

1D modeling tools like Dymola has a variety of libraries such as controller, hydraulics, electrics and so forth, and it is possible to create simple holistic system model. Simulation with simple system model is suitable for the early phase of development and reducing cost.

FMI is a generic interface to connect different modeling tools that provides more flexible simulation environment. In addition, FMI is useful for model transfer between parts supplier and OEM or across different simulation domains and it is promising to spread widely in future.

In this paper, we built Excavator model consisting of hydraulic controller and mechanics parts. We used Simpack to model mechanics parts and Dymola for hydraulic controller. We generated FMU on hydraulic controller from Dymola and coupled it with Simpack, via both Co-simulation and Model Exchange to see the difference of those two approaches in light of simulation accuracy as well as calculation time. Then we developed an optimization workflow with Isight to determine controller parameters to see if the approach is effective for the early conceptual phase of product development.

\section{Excavator Model}

Figure 1 shows structure of Excavator system model. 


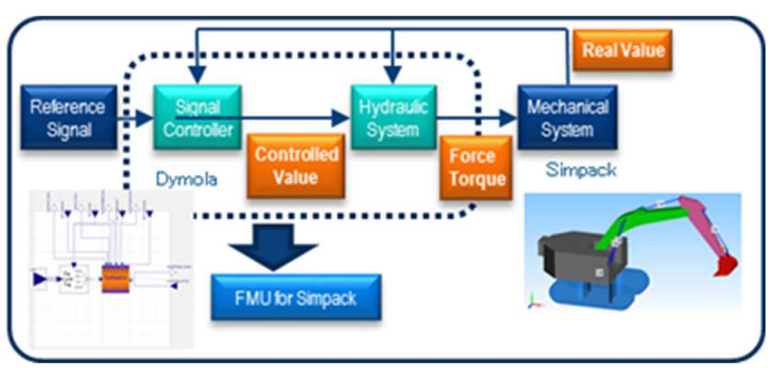

Figure 1. Structure of excavator system model

The Excavator model consists of two parts, mechanics and hydraulic controller. Mechanics parts are constructed by Multi-body simulation software Simpack and hydraulics controller is created with 1D Simulation tool Dymola.

Simpack and Dymola are connected by FMI. Simpack outputs real value of each actuator cylinder length, and Dymola outputs force and torque value of each actuator through FMI. We developed this Excavator model based on publicly available OpenHydraulics by Georgia Institute of Technology.

\subsection{Vehicle Model}

Figure 2 shows the mechanics parts model.

The model is constructed by Simpack. All parts (Base, Carriage, Boom, LinkArm, and Bucket) are defined as Rigid Body. The Rigid body has a mass, inertia, center of gravity position.

Each body parts have degrees of freedom on connecting point by Joints and Constraints element in Simpack.

Each actuator is modelled by Force element in Simpack. Actuator between Base and Carriage is defined as torque around normal direction (y). Cylinder actuator of Boom, LinkArm, and Bucket are defined as translational force. In addition, Boom is equipped with two actuators on the left and right. All value of actuator force and torque are provided from hydraulic controller in Dymola via FMI.

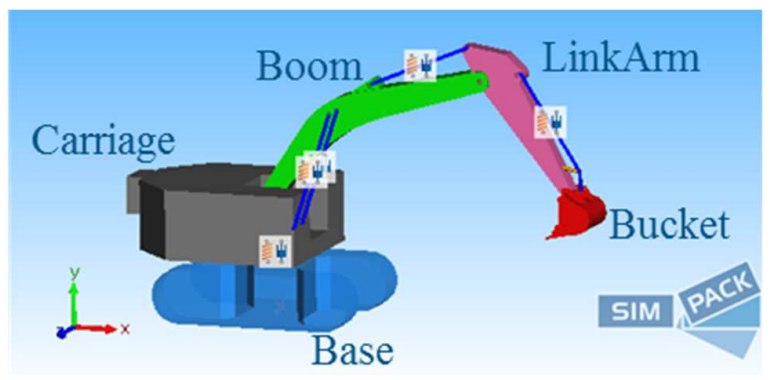

Figure 1. Vehicle Model (Simpack)

\subsection{Hydraulics model}

Figure 3 and Figure 4 shows the hydraulic and controller model modeled in Dymola.

Hydraulic consist of Power Unit, Valve Unit and cylinder model. Controller gives control values to Valve Unit.

Controller model shown in Figure 4 gives control value calculated by PI control differential of real cylinder length and target length to Valve unit.

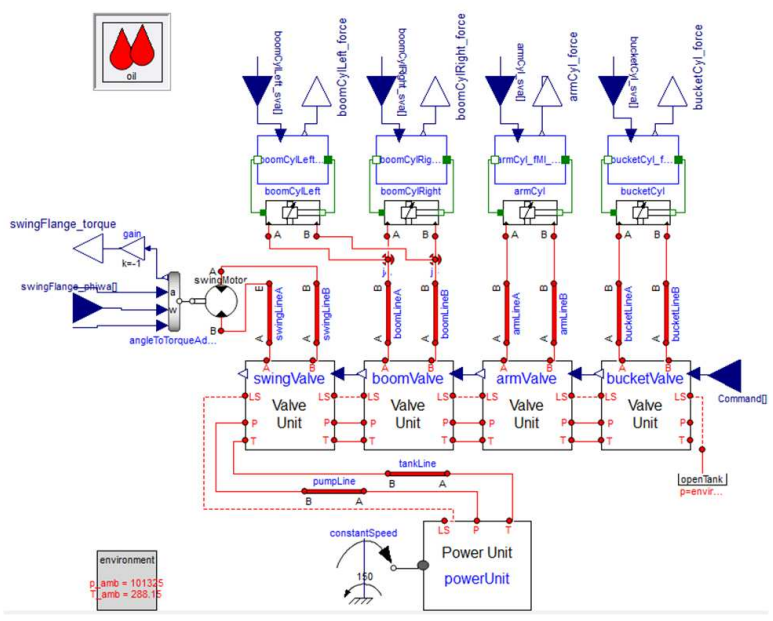

Figure 3. Hydraulics Model (Dymola)

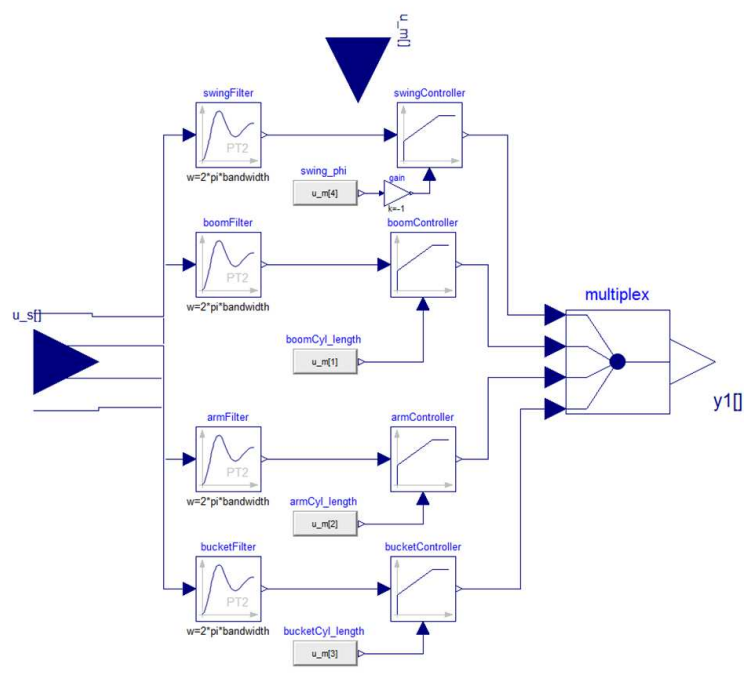

Figure 4. Controller Model (Dymola)

To simulate whole Excavator system creating integrate model using FMI, to connect mechanics and hydraulics controller models created in different modeling tools.

In this case, hydraulic controller model is converted to FMU in Dymola and integrate it to Simpack model. We used version 2.0 of FMU. 


\section{Comparison of Co-Sim and ME}

There are two types of FMU, Co - Simulation and Model Exchange, which use different solvers. Co Simulation uses solver included in each FMU, and sets communication time between tools/models and execute iteration step for each. In this case, the communication time was set to $0.1 \mathrm{~ms}$ in Co-Simulation. On the other hand, in Model Exchange FMU contains only models and not the solver, and the calculation is done by the solver on the simulation platform. In our case, CoSimulation executes both Dymola and Simpack solver and Model Exchange executes only Simpack solver.

Optimization calculation requires a lot of iteration, so, it is desirable to have shorter simulation time. To see the impact of two different FMU modes onto the calculation time, we executed both Co-Simulation and Model Exchange FMU cases and compared the calculation result and calculation time.

As simulation scenario, we defined following steps shown as Figure 5.

1) Vehicle speed is 0 at all steps.

2) Extend Boom and LinkArm

3) Scoop sand

4) Lift Bucket

5) Turn Carriage

6) Dump sand

We simulate only the behavior of the working machine and do not consider the behavior of sand and ground.

In order to perform this series of operations, we set target cylinder length as time domain data, and gave force via feedback control. Figure 6 shows the comparison results between Co-Simulation and Model Exchange taking Boom cylinder length, and Figure 7 takes the difference of Boom cylinder length between two FMU approaches.

From Figure 6, we see that the results of CoSimulation and Model Exchange are almost same. Figure 7 shows the difference between these two and it is smaller than the actual values by three orders of magnitude so we can consider that these two FMU approaches brought almost the same result.

In Figure 6, there is a difference between the target value and the calculation result. However, in this case, since temporary control parameters are used so accuracy of target value was not reviewed.

Regarding comparison of the computation time in Table 1, calculation time of Model Exchange was 1/20 from Co-Simulation. Therefore, we decided using
Model Exchange for the subsequent optimization calculation.

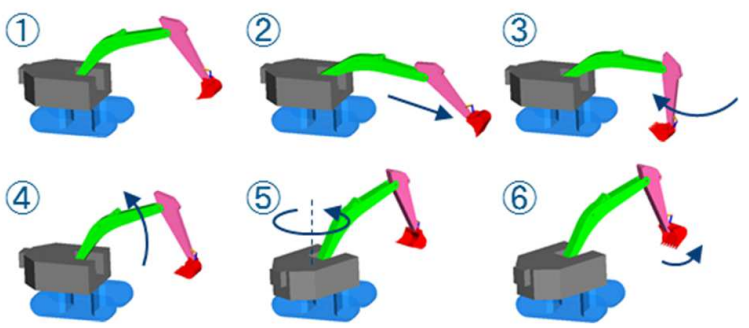

Figure 5. Calculation step

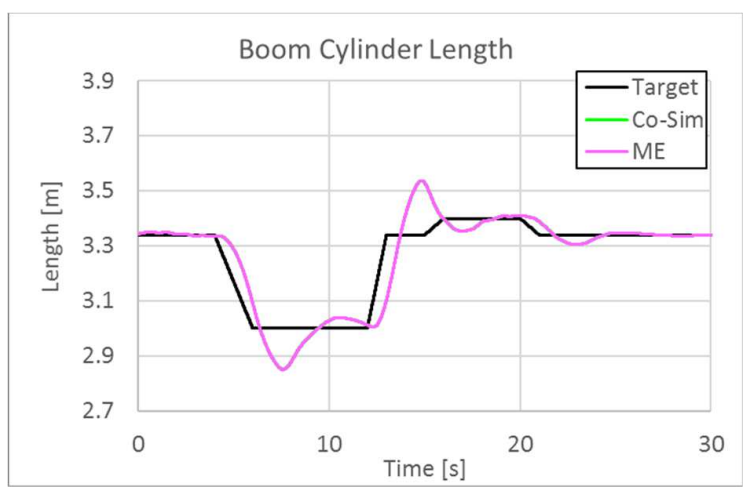

Figure 6. Boom Cylinder length (Co-sim vs ME)

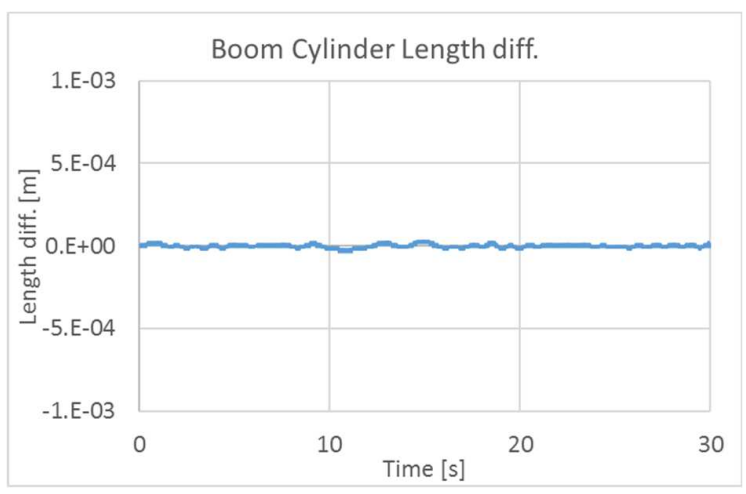

Figure 7. Difference of Boom Cylinder length between Co-simulation and model Exchange.

Table 1. Sizes of Compiler Phases, Lines of Code.

\begin{tabular}{|l|r|c|}
\hline FMU Type & Calculation Time & CPU Time \\
\hline Co-Sim & $46 \mathrm{~m} 42 \mathrm{~s}$ & $95.09 \mathrm{~s}$ \\
\hline ME & $1 \mathrm{~m} 43 \mathrm{~s}$ & $28.46 \mathrm{~s}$ \\
\hline \multicolumn{2}{|c|}{ ※CPU : Intel®Core ${ }^{\mathrm{TM}}$ i7 $2.7 \mathrm{GHz} 、$ Momory:16.0GB }
\end{tabular}

In the comparison of CPU Time, there is no difference as much as calculation time, so we consider that Co-Simulation took longer calculation time than 
Model Exchange because the communication time between tools is an important factor.

\section{Control Parameters Optimization}

We use the coupled model using FMU to explorer design parameters for control.

In this study, as an example of the control robustness against the difference in the operating environment of the working machine, we optimized control parameters for two cases with different Bucket mass parameters.

The operating conditions are the same as the series of operations shown in Figure 5.

Table 2 outlines the optimization calculation.

Table 2. Optimization Outline

\begin{tabular}{|l|l|}
\hline Bucket Condition & $\begin{array}{l}\cdot \text { Payload off } \\
\cdot \text { Payload } 50 \mathrm{~kg}\end{array}$ \\
\hline Design variable & $\begin{array}{l}\text { Controller gain and time } \\
\text { constant for each cylinder } \\
\text { (Total 6 parameter) }\end{array}$ \\
\hline Objective & $\begin{array}{l}\text { Minimize cylinder } \\
\text { length error } \varepsilon\end{array}$ \\
\hline
\end{tabular}

We set the error $\varepsilon$ between the objective and the target value of cylinder length as following equation.

$$
\varepsilon=\sqrt{\frac{1}{n-1} \sum_{n=1}^{n}\left(y_{\text {real }}-y_{o b j}\right)^{2}}
$$

In the design exploration, we ran DOE calculation at the beginning to create an approximate model from the result. DOE was performed by Latin Hypercube, and approximate model was created using RBF (Radial Basis Function). Optimization was carried out to minimize the error defined by the equation above using the created approximate model. The optimization method was NCGA (Neighborhood Cultivation Genetic Algorithm). These workflows were created using Isight. Figure 8 shows the outline of the design search.

Figure9 shows the comparison of approximate model and actual model. The approximate model is created based on DOE results by RBF method. The result of approximate model is in good agreement with the result of actual model, so the approximate model can be applied to optimization.

Figure 10 to Figure 13 show the calculation results.
Figure 10 and Figure 11 show the time domain data of the difference with respect to the target value of the Boom cylinder length, and Figure 12 and Figure 13 show the time domain data of the target value and the calculated cylinder length.

As can be seen from Figure 10 and Figure 11, the error with respect to the target value becomes smaller by using the optimized parameters.

We can also confirm the effectiveness of the optimization by comparing the cylinder lengths in Figure 12 and Figure 13.
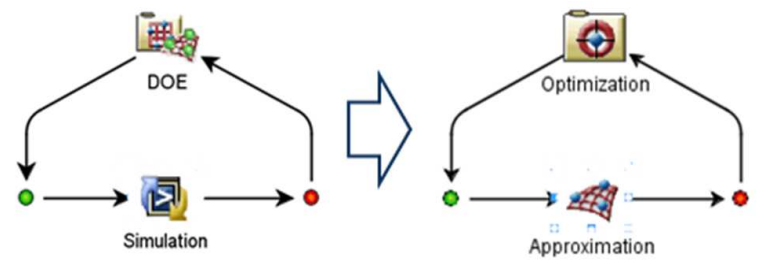

Figure 8. Workflow of optimization in Isight

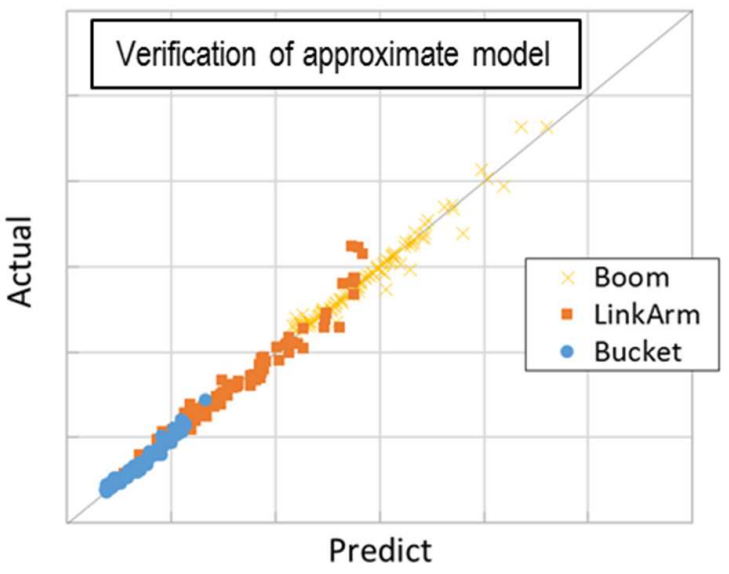

Figure 9. Verification result of approximate model

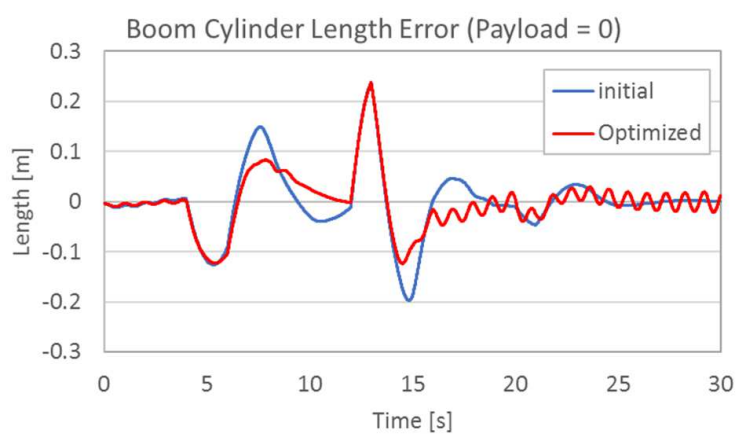

Figure 10. Boom Length Error (Payload off) 


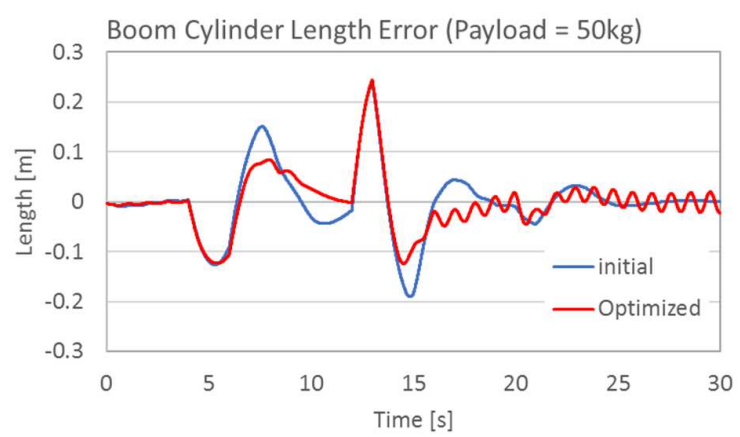

Figure 11. Boom Length Error (Payload 50kg)

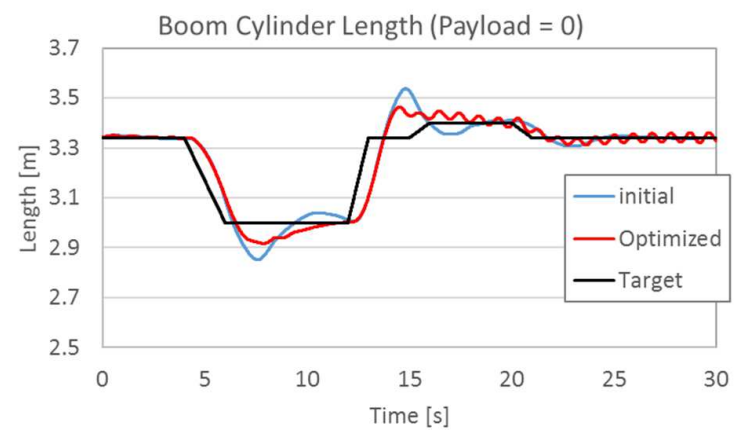

Figure 12. Boom Length (Payload off)

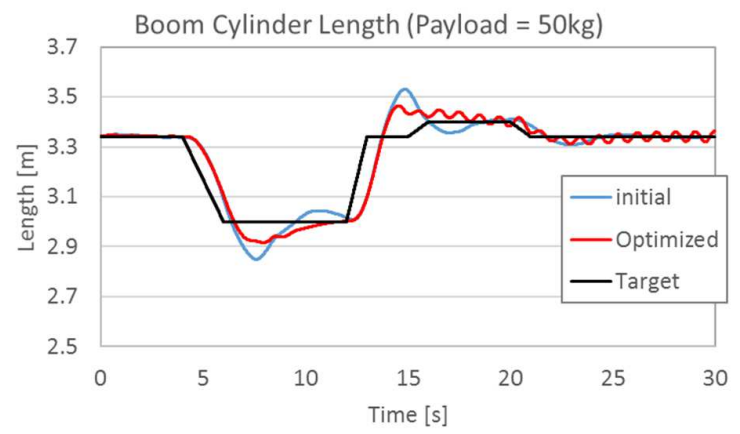

Figure 13. Boom Length (Payload 50kg)

\section{Conclusion}

For a construction machine, we modeled a system including hydraulic controller with 1D simulation tool and mechanism analysis software and created an integrated simulation model using FMU.

In this case, it turned out that FMU Model Exchange worked efficiently for the optimization of control parameters.

In this study, we performed design space exploration on control parameters, but we think that it can be applied not only to control parameters but also to dimensioning components such as the shape of structural parts.

As a conclusion, we consider that the use of the system model with FMU is effective for the design search at the early stage of development where we need to evaluate systems performance coupling different disciplines efficiently.

\section{Acknowledgements}

We gratefully acknowledge the support of $\mathrm{Mr}$. Youngwon HAHN from Dassault Systemes Simulia Corp for his great cooperation in technical advice and model creation for this thesis.

\section{References}

Dassault Systems, Dymola User Manual Volume1,2

Version 2018 (2017-04-10)

Dassault Systemes Simulia Corp., Simpack Documentation Version 2018

Georgia Institute of Technology, OpenHydraulics v1.0.1 (2013-02-26)

https://github.com/cparedis/OpenHydraulics 